\title{
Forum
}

\section{Die Berner Psychosenmatinée oder «Ein Versuch der institutionsübergreifenden Verständigung zum Thema der Psychose 2009-2011»}

\author{
Hans-Rudolf Schneider (Zürich)
}

Seit 2009 treffen sich in unregelmässigen Abständen vier PsychoanalytikerInnen unterschiedlicher theoretischer und institutioneller Zugehörigkeit (Elisabeth Aebi Schneider, Schweiz. Gesellschaft für Psychoanalyse, Bern; Liliane Schaffner, Psychoanalyse am Werk, Bern; Hans-Rudolf Schneider, Psychoanalytisches Seminar Zürich, Zürich; Christoph Zimmermann, Psychoanalyse am Werk, Bern), jedoch mit einem gemeinsamen Interesse an theoretischer und praktischer Auseinandersetzung mit psychotischen Patienten, um ihre Gedanken zu theoretischen, praktischen und technischen Fragen auszutauschen. Elisabeth Aebi Schneider und Hans-Rudolf Schneider hatten vorgängig mehrere Jahre als Dozierende an der Münchner Weiterbildung in Psychoanalytischer Psychosentherapie mitgearbeitet und in Bern eine Supervisionsgruppe für KollegInnen, die mit psychotischen Patienten und Patientinnen arbeiten, geführt. Das immer wieder geäusserte Interesse an einem Austausch von Erfahrungen mit der Behandlung psychotischer Patienten ermutigte uns in der Vierergruppe, nach einer Form zu suchen, welche die Öffnung der Diskussion für ein weiteres am Thema «Psychoanalyse der Psychose» interessiertes Fachpublikum erlauben könnte. Erstmals luden wir am Sonntag, dem 22. November 2009, zu einer von uns so genannten Psychosenmatinée nach Bern ein und haben seither jeweils im November einmal jährlich ein thematisch umschriebenes Treffen zum Thema Psychosen organisiert.

Im Verlauf des Jahres wählen wir in der Regel zwei Texte zum Thema aus, je ein Text lacanianischer und einer klassisch freudianischer Provenienz. Den angemeldeten Teilnehmenden werden die Texte vorgängig zugestellt, damit sie sich auf 
die Tagung vorbereiten und so leichter eigene Ideen oder spezifische Fragen in die Diskussion einbringen können. Zu jedem Text verfassen die vier OrganisatorInnen je einen kurzen Input, den sie zu Beginn der Tagung vortragen. Das dient dem Anstoss der angestrebten Diskussion unter den Anwesenden.

Der ersten Matinée stellten wir Freuds Zitat aus den «Konstruktionen in der Analyse» voran: «Ich glaube nicht, dass diese Auffassung des Wahns vollkommen neu ist, aber sie betont doch einen Gesichtspunkt, der für gewöhnlich nicht in den Vordergrund gerückt wird. Wesentlich an ihr ist die Behauptung, dass derWahnsinn nicht nur Methode hat, wie schon der Dichter erkannte, sondern dass auch ein Stück historischer Wahrheit in ihm enthalten ist, und es liegt uns nahe anzunehmen, dass der zwanghafte Glaube, den derWahn findet, gerade aus solch infantiler Quelle seine Stärke bezieht. Mir stehen heute, um diese Theorie zu erweisen, nur Reminiszenzen zu Gebote, nicht frische Eindrücke. Es würde wahrscheinlich der Mühe lohnen, wenn man versuchte, entsprechende Krankheitsfälle nach den hier entwickelten Voraussetzungen zu studieren und auch ihre Behandlung danach einzurichten» (Freud, G.W., XVI, S.54-55).

Wir wählten Texte von Alain Gibeault (2004)«Therapeutische Strategie und Deutungstaktik in der Psychose» und von Thomas Vogt (2007) «Verliebt-Verkannt - Verrückt» aus, eine Auswahl, die uns sogleich in die spannende Diskussion der unterschiedlichen psychoanalytischen Theoretisierung der Psychose führte.

Gibeault, der in seiner Publikation von einer für die Arbeit mit psychotischen Patienten entwickelten spezifischen Form des psychoanalytischen Psychodramas ausgeht, liess uns über die Probleme der Technik und der dieser zu Grunde liegenden mehr oder weniger expliziten Theorie der Psychose nachdenken. Die von ihm beschriebenen Charakteristika psychotischer Struktur wie Spaltung, Verleugnung, Schwierigkeiten in der synthetischen Funktion und die Formen der Intervention weckten bei den Lacanianern kritische Stimmen und führten so zu einer intensiven Diskussion theoretischer und technischer Fragen, bei denen die Möglichkeiten der Arbeit an den Repräsentanzen, oder anders gesagt die Symbolisierung, in ihrer Bedeutung diskutiert wurde. Vogt seinerseits brachte mit seinem lacanianischen Ansatz die Fragen der Grenze der Therapie, der Differenz psychotischer und nichtpsychotischer Strukturen und der prinzipiellen Veränderbarkeit einer psychischen Struktur aufs Tapet.

Es zeigte sich in der Folge, dass diese zentralen Themen uns auch in den nächsten Psychosenmatinéen immer wieder beschäftigen würden, genauso wie die Problematik der Indikation, die unweigerlich von den jeweiligen Konzepten und den unterschiedlich eingeschätzten Möglichkeiten psychoanalytischer Behandlung 
bestimmt wird. Kurz wurde gestreift, dass der Psychoanalytiker, der mit psychotischen Menschen arbeitet, über spezifische Voraussetzungen verfügen muss, wie beispielsweise die von Racamier beschriebene narzisstische Verführbarkeit.

Es bewährte sich für die Diskussion sehr, dass wir für die erste Psychosenmatinée Texte ausgewählt hatten, die sich anhand ausführlich dargestellter klinischer Beispiele mit Theorie und Technik auseinandersetzen. Dies regte an, auf eigene Erfahrungen zurückzugreifen und erleichterte es, die differenzierten und oftmals nicht leicht verständlichen theoretischen Überlegungen der Autoren nachzuvollziehen.

In den zwei folgenden Jahren diskutierten wir Texte, die sich je mit einem umschriebenen Thema zur Problematik der Psychoanalyse der Psychosen beschäftigen. 2010 wählten wir das anspruchsvolle, zentrale Thema der «Übertragung in der Psychosenbehandlung» und stellten der Tagung erneut ein Zitat Freuds voran: «Der Eremit kehrt dieser Welt den Rücken, er will nichts mehr mit ihr zu tun zu haben. Aber man kann mehr tun, man kann sie umschaffen wollen, anstatt ihrer eine andere aufbauen, in der die unerträglichsten Züge ausgetilgt und durch andere im Sinne der eigenen Wünsche ersetzt sind. Wer in verzweifelter Empörung diesen Weg zum Glück einschlägt, wird in der Regel nichts erreichen; die Wirklichkeit ist zu stark für ihn. Er wird ein Wahnsinniger, der in der Durchsetzung des Wahns meist keine Helfer findet. Es wird aber behauptet, dass jeder von uns sich in irgendeinem Punkte ähnlich wie der Paranoiker benimmt, eine ihm unleidliche Seite der Welt durch eine Wunschbildung korrigiert und diesen Wahn in die Realität einträgt.» (Freud, GW, XIV, S 439-440).

Als Ausgangspunkt für die Diskussion dienten drei äusserst anregende Publikationen: Michael Turnheims Arbeiten (1996) «Der Mechanismus der Psychose» und «Übertragung und Psychose» vertieften die Fragen, die uns im ersten Jahr sehr beschäftigt hatten, und sie stellten gleichzeitig dar, wie ein Lacanianer Freud neu liest. Evelyne Kestembergs Arbeit (2001) «Die FetischObjektbeziehung» gab Gelegenheit, sich mit einem zentralen Konzept der französischen Psychoanalytikerin auseinanderzusetzen, die sich intensiv mit Psychotikern und Adoleszenten beschäftigt hat. Dabei grenzt sie die beschriebene Form der Objektbeziehung von den eigentlichen Übertragungen ab. Ihre Arbeit führte zu einer differenzierten Betrachtung der verschiedenen Bedeutungen der Sprache und des Sprechens sowie des Fetisch mit seinen möglichen Beziehungen zum Sinthome. In den Arbeiten von Turnheim und bei deren Diskussion tauchte die Frage der sexuellen Identität respektive der Sexuierung auf, was uns veranlasste, die Tagung im November 2011 dem Thema «Psychose und Weiblichkeit» zu wid- 
men. Auch diesmal bot sich ein Zitat von Freud als Motto an, diesmal aus der 33. Vorlesung:

«Über das Rätsel der Weiblichkeit haben die Menschen zu allen Zeiten gegrübelt: Häupter in Hieroglyphenmützen, Häupter in Turban und schwarzem Barett, Perückenhäupter und tausend andere arme, schwitzende Menschenhäupter... (Heine, Nordsee). Auch Sie werden sich von diesem Grübeln nicht ausgeschlossen haben, insoferne Sie Männer sind; von den Frauen unter Ihnen erwartet man es nicht, sie sind selbst dieses Rätsel.» (Freud, GW, XV, S. 120).

Die zwei eindrücklichen und ausführlichen Falldarstellungen von Brigitte Reed-Duvaille (2011) «Salomé ou Comment j’ai tué ma mère». Psychose et trouble de l'identification féminine und von Martine Lerude (1994) «D'une psychose féminine» forderten sowohl die OrganisatorInnen wie auch die Teilnehmenden der Matinée, ging es doch einmal mehr darum, die sehr unterschiedlichen Ansätze der Lacanianer und der klassischen Freudianer deutlich werden zu lassen, Differenzen nicht zu überspielen, jedoch zu versuchen, den andern in seinen theoretischen Ansichten etwas besser zu verstehen. Übereinstimmung und scheinbar unüberbrückbare Differenz der Standpunkte bestimmten in dauerndem Wechsel die Diskussion, die aber stets interessiert und freundschaftlich verlief. Wie schon an den vorangegangenen Tagungen verstand man sich am besten in der Klinik. Allmählich wurde aber auch deutlich, wie sich bei allen Beteiligten ein respektvolles gegenseitiges Verständnis etlicher schwieriger Aspekte der theoretischen Ansichten und Haltungen Platz geschaffen hatte-wohl nicht zuletzt dank der jetzt doch schon mehrere Jahre dauernden Zusammenarbeit. Zum ersten Mal wurde diesmal ein kurzer Ausschnitt aus einem Film zur Illustration des Vorgetragenen gezeigt: In einer Sequenz aus "Pirates of the Caribbean” zieht die verführerische Meerjungfrau die Männer in ihren Booten ins Verderben. Die Weiblichkeit als psychotische Bedrohung? Wie unterschiedlich die Überlegungen zur Behandlung psychotischer PatientInnen diskutiert wurden, lässt sich an der aufgeworfenen Frage ersehen, ob es denn nicht bei den Psychotikern um die Herstellung einer Identität mit Hilfe von Identifikationen beispielsweise mit der Analytikerin, oder dem Analytiker also schliesslich um die Herstellung eines phallischen Scheins gehe, während andere die Ermöglichung einer verbesserten Symbolisierungsfähigkeit und damit die Arbeit an den Repräsentanzen in das Zentrum der Therapie stellten.

Die dritte Psychosenmatinée stiess erneut auf grosses Interesse und regte auch uns Organisierende sehr an, so dass wir beschlossen, die Reihe in der bisherigen Form weiter zu führen. 
Wenn immer möglich sollen die bisherigen Grundsatztexte sowie unsere Diskussionsbeiträge alle drei Jahre in einer einfachen Form publiziert werden.

Nächste Psychosenmatinée: 25. November 2012.

Auskunft für Interessierte: elaebi@postmail.ch.

\section{Literatur}

Freud, Sigmund (1930). Das Unbehagen in der Kultur, G.W., XIV, S. 439 - 440.

Freud, Sigmund (1933). Die Weiblichkeit, 33. Vorlesung, G.W. XV, S. 120.

Freud, Sigmund (1937). Konstruktionen in der Analyse, G.W., XVI, S. 54 - 55.

Gibeault, Alain (2004). Stratégie thérapeutique et tactique interprétative dans le travail psychanalytique avec les patients psychotiques (Therapeutische Strategie und Deutungstaktik in der Psychose), Vortrag an der 22. Jahrestagung der EPF, Brüssel, 2004.

Kestemberg, Evelyne (2001). La relation fétichique à l’objet, in: Kestemberg, E., La psychose froide, PUF, Paris (Deutsch: «Die Fetisch-Objektbeziehung», in: Forum der Psychoanalytischen Psychosentherapie, 15, Vandenhoeck \& Ruprecht, Göttingen, 2006).

Lerude, Martine (1994). D’une psychose féminine, In: Fondation Européenne pour la Psychanalyse, 1958 - 1993, L’abord des psychoses après Lacan, Bordeaux.

Reed-Duvaille, Brigitte (2011). Salomé ou «Comment j’ai tué ma mère». Psychose et trouble de l'identification féminine, In: Identité et sentiment d'existence, Psychanalyse et Psychoses, Paris, 11.

Turnheim, Michael (1996). Der Mechanismus der Psychose, und: Übertragung und Psychose, beide in: Turnheim, M., Versammlung und Zerstreuung, Psychoanalytische Aufsätze II, Turia \& Kant, Wien.

Vogt, Thomas (2007). Verliebt - Verkannt - Verrückt, in: Widmer/Schmid (Hg), Psychosen - eine Herausforderung für die Psychoanalyse. Strukturen-KlinikProduktionen. Transkript, Bielefeld. 